\title{
Epiphyseal lesion of the humerus in a child
}

\author{
Eric R. Henderson • Rebecca A. Pohlmann • \\ Monica L. Hollowell • Mark C. Gebhardt
}

Published online: 10 January 2013

(C) ISS 2013

\section{History}

A 13-year-old boy presented with a one-and-a-half-month history of right shoulder pain that was precipitated by a competitive wrestling episode during which he felt a "cracking" sensation. The patient did not seek immediate medical attention and was treated at home with a sling, ice, and ibuprofen. After incomplete pain relief radiographs were obtained (Fig. 1). Past medical history was unremarkable. Physical examination revealed a right upper extremity that had no muscular asymmetry, normal neurological and vascular function, and normal motor function with a full range of shoulder motion in all planes and axes.

A magnetic resonance imaging (MRI) scan followed by open biopsy were performed (Figs. 2 and 3).

What is the diagnosis?
Fig. 1 AP-external rotation (a) and AP-internal rotation (b) radiographs of the right proximal humerus
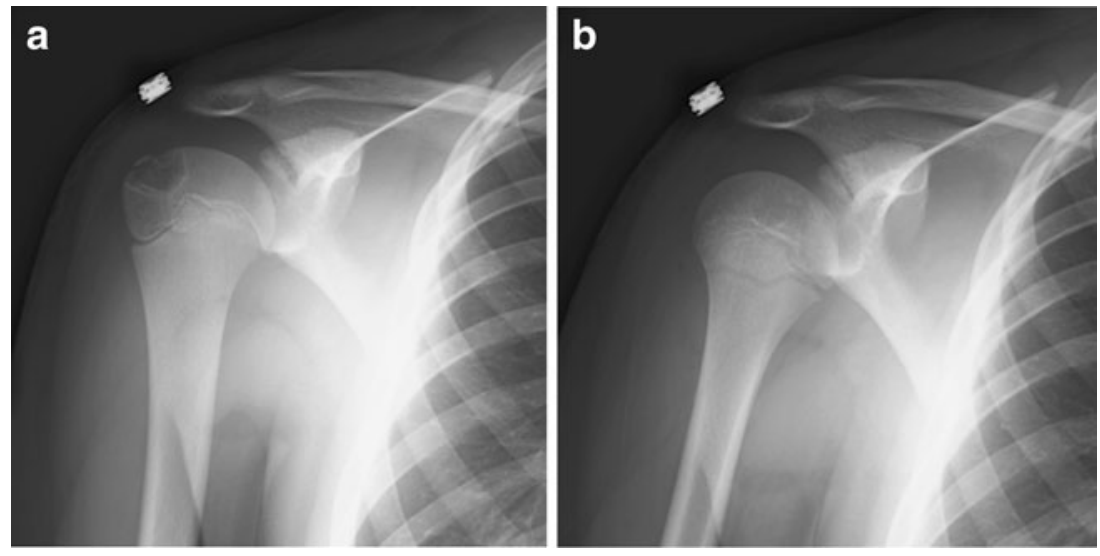

The diagnosis can be found at doi: 10.1007/s00256-012-1567-2

E. R. Henderson $(\bowtie)$

Division of Orthopaedic Oncology, Department of Orthopaedic Surgery, Norris Cotton Cancer Center, Dartmouth-Hitchcock

Medical Center, The Geisel School of Medicine at Dartmouth College, One Medical Center Drive,

Lebanon, NH, USA

e-mail: eric.r.henderson@gmail.com

\section{R. A. Pohlmann}

Department of Pathology, Beth Israel Deaconess Medical Center, Harvard Medical School, Boston, MA, USA
M. L. Hollowell

Department of Pathology, Boston Children's Hospital, Harvard Medical School, Boston, MA, USA

M. C. Gebhardt

Department of Orthopaedics, Beth Israel Deaconess Medical Center, Harvard Medical School, Boston, MA, USA 
Fig. 2 a T1-weighted coronal image (repetition time/echo time, 734/21). b T2-weighted coronal image with fat suppression (repetition time/echo time, $5,320 / 78)$

Fig. 3 Low-power $(\times 4, \mathrm{H} \& \mathrm{E})$ histological image (a); lowpower $(\times 20, \mathrm{H} \& \mathrm{E})$ histological image (b)
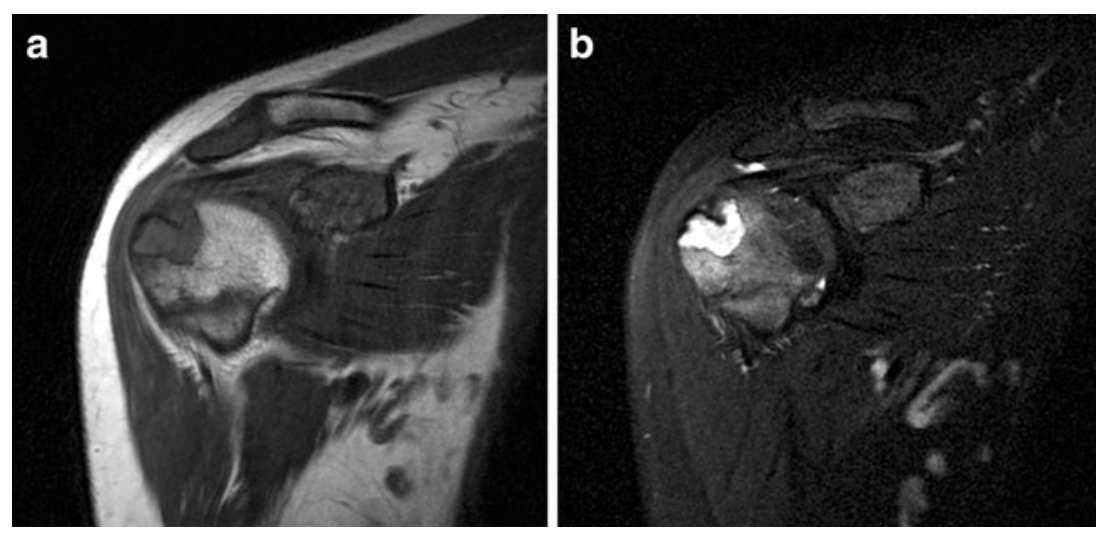

\section{a}
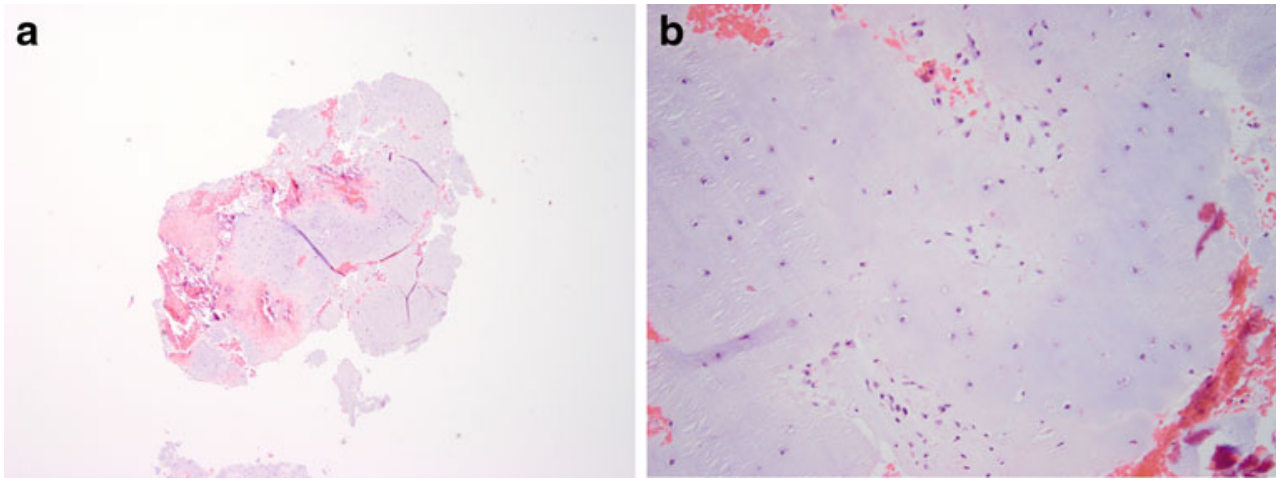